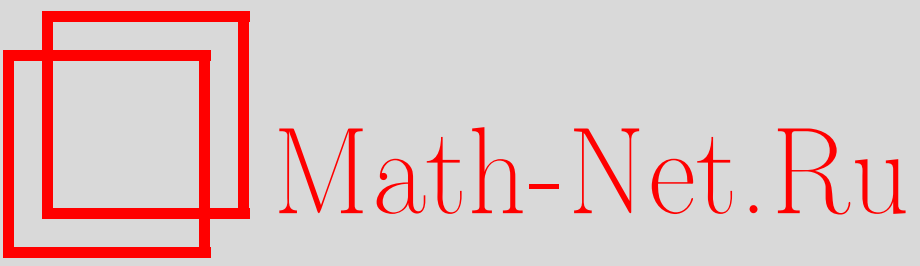

М. Б. Губайдуллин, О свойствах спектра оператора Шредингера в магнитном поле, ТМФ, 2002, том 130, номер 2, 267-274

DOI: https://doi.org/10.4213/tmf301

Использование Общероссийского математического портала Math-Net.Ru подразумевает, что вы прочитали и согласны с пользовательским соглашением

http://www.mathnet.ru/rus/agreement

Параметры загрузки:

IP: 34.229 .108 .108

26 апреля 2023 г., 15:14:25 


\section{О СВОЙСТВАХ СПЕКТРА ОПЕРАТОРА ШРЕДИНГЕРА В МАГНИТНОМ ПОЛЕ}

Исследованы свойства спектра оператора Шредингера с магнитным и электрическим потенциалами. Доказаны отсутствие у оператора положительных собственных значений и абсолютная непрерывность спектра этого оператора на положительной полуоси.

\section{1. ВВЕДЕНИЕ}

В работе изучается оператор Шредингера в пространстве $L_{2}\left(R^{n}\right)$

$$
H(a, V)=\sum_{k=1}^{n}\left(p_{k}+a_{k}\right)^{2}+V,
$$

где $p_{k}=-i \partial / \partial x_{k}$ - оператор импульса по $k$-й переменной, $V(x)-$ электрический потенциал, $a(x)=\left(a_{1}(x), \ldots, a_{n}(x)\right)$ - магнитный потенциал, $a_{k}(x) \in W_{2, \text { loc }}^{2}$, причем все потенциалы считаются вешественными. Данную работу можно рассматривать как продолжение статьи [1].

\section{2. ОТСУТСТВИЕ ПОЛОЖИТЕЛЬНЫХ СОБСТВЕННЫХ ЗНАЧЕНИЙ}

С физической точки зрения утверждение о том, что у оператора (1) (при некоторых разумных ограничениях на потенциалы) отсутствуют положительные собственные значения, представляется очевидным, поскольку положительным собственным значениям (1) соответствуют связанные состояния с положительной энергией связи. Однако известен пример электрического потенциала Вигнера-фон Неймана $(a(x) \equiv 0)$, который ведет себя на бесконечности как $O(1 /|x|)$ и оператор (1) которого имеет положительное собственное значение. В теореме 1 приводятся условия на потенциалы, исключающие такую возможность. Эта теорема является обобшением теоремы 2.2 работы [2], которая была доказана для случая $a(x) \equiv 0$. Из вида оператора (1) следует, что

$$
e^{-i \mu} H(a, V) e^{i \mu}=H(b, V),
$$

\footnotetext{
* Башкирский государственный университет, Уф̆а, Россия
} 
где $\mu(x)$ - произвольная вешественная дифференцируемая функция, назьваемая калибровочной. В случае специального выбора $\mu(x)$ (см. [1]) имеем

$$
b_{k}(x)=\sum_{m=1}^{n} x_{m} \int_{0}^{1} s a_{k m}(s x) d s, \quad k=\overline{1, n}
$$

где $b_{k}(x)$ - компоненты нового магнитного потенциала, $a_{k m}(x)=\partial a_{k}(x) / \partial x_{m}-$ $\partial a_{m}(x) / \partial x_{k}-$ компоненты магнитного тензора. Введем величину

$$
\hat{b}_{k}(x)=\sum_{m=1}^{n} \frac{x_{m}}{|x|} a_{k m}(x), \quad k=\overline{1, n}
$$

тогда

$$
\frac{\partial b_{k}(x)}{\partial|x|}=-\frac{b_{k}(x)}{|x|}+\hat{b}_{k}(x), \quad k=\overline{1, n} .
$$

ТЕОрема 1. Пусть $H(a, V) U=\lambda U, \lambda>0$ и пусть выполнены следующие условия:

a) $\left|\hat{b}_{k}(x)\right|=o\left(\frac{1}{|x|}\right), \quad|x| \rightarrow \infty, \sup _{x \in R^{n}}\left|\hat{b}_{k}(x)\right|<\infty, \quad k=\overline{1, n}$;

б) $\sup _{x \in R^{n}}|\operatorname{div} b(x)|<\infty$;

в) при $n \leqslant 3$ оператор $W=V(1+|x|) \Delta$-компактен;

г) при $n>3$ оператор $W \Delta$-компактен $и$

$$
\lim _{z \rightarrow+\infty} z^{\frac{1}{4}}\left\|(1+|x|)^{-\frac{1}{2}} W(-\Delta+z)^{-1}\right\|=0 .
$$

Тогда $U(x) \equiv 0$.

Сделаем некоторые предварительные замечания. Условия "а" и “б” выполнены, если

$$
\left\{\begin{array}{l}
\lim _{|x| \rightarrow+\infty}|x| a_{k m}(x)=0, \quad \sup _{x \in R^{n}}\left|a_{k m}(x)\right|<\infty, \quad k, m=\overline{1, n} \\
\left|\frac{\partial a_{k m}(x)}{\partial x_{k}}\right| \leqslant \frac{C}{1+|x|}, \quad k, m=\overline{1, n}
\end{array}\right.
$$

Здесь и далее $C$ - любая подходяшая абсолютная постоянная. Налагать условия непосредственно на компоненты магнитного потенциала $a_{k}(x)$ нет необходимости из-за их отсутствия в наших формулах. В качестве электрических потенциалов допускаются потенциалы вида $V(x)=(1+|x|)^{-1} W(x)$, где $W(x)$ - произвольная (не обязательно гладкая) функция такая, что $\sup _{x \in R^{n}}|W(x)| \leqslant C$ и $\lim _{|x| \rightarrow+\infty}|W(x)|=0$. При этих условиях оператор $W \Delta$-компактен и, кроме того, $\left\|W(-\Delta+z)^{-1}\right\| \leqslant C z^{-1}$, так что и предельное условие “г” выполнено. Если функция $W(x)$ неограниченна, то при $n \leqslant 3$ критерий $\Delta$-компактности $W$ хорошо известен и заключается в том, что

$$
\lim _{|x| \rightarrow+\infty} \int_{|x-y| \leqslant 1}|W(y)|^{2} d y=0 .
$$


При этом для таких потенциалов предельное условие “г” выполняется автоматически [1]. В случае, когда $n \geqslant 4$, условие “г” будет выполнено, если

$$
\left\{\begin{array}{l}
\lim _{x \rightarrow+\infty} \int_{|x-y| \leqslant 1}|x-y|^{-n+3}|W(y)|^{2} d y=0, \\
\lim _{\gamma \rightarrow 0} \sup _{x \in R^{n}} \int_{|x-y| \leqslant \gamma}|x-y|^{-n+3} \frac{|W(y)|^{2}}{1+|y|} d y=0 .
\end{array}\right.
$$

При этом первое условие обеспечивает $\Delta$-компактность $W$, а второе - вьполнение предельного условия "г".

Для доказательства теоремы 1 используется идея метода, предложенного в книге [3] (c. 102). Однако предлагаемое ниже доказательство представляется более простым, поскольку использует характерную для эллиптических уравнений априорную оценку.

ДокАЗАТЕЛЬСтво. Как и в работе [1], определим пространства $L_{2}\left(R_{+}, G\right), G=$ $L_{2}\left(S_{n-1}, d \omega\right)$, где $S_{n-1}$ - единичная сфера, $\omega=x /|x|,|x|=r$. Обозначим через \|\|$_{G}$ норму в $G$. Между элементами пространств $L_{2}\left(R^{n}\right)$ и $L_{2}\left(R_{+}, G\right)$ можно установить взаимно однозначное соответствие, сохраняющее норму. Далее в этом разделе под символами $(.,),. \|$. \| будут пониматься скалярное произведение и норма в пространстве $L_{2}\left(R_{+}, G\right)$, соответственно. Согласно лемме 4 из работы [1] имеем

$$
\widetilde{H}(b, V)=-\frac{\partial^{2}}{\partial r^{2}}-\frac{n-1}{2 r^{2}}+\frac{1}{r^{2}} \sum_{k=1}^{n} D_{k}^{2}+\frac{1}{r} \sum_{k=1}^{n}\left(D_{k} b_{k}+b_{k} D_{k}\right)+b^{2}+V,
$$

где $\widetilde{H}(b, V)$ - оператор в $L_{2}\left(R_{+}, G\right)$, соответствуюший оператору $H(b, V)$ в $L_{2}\left(R^{n}\right), D_{k}$, $k=\overline{1, n},-$ симметрический оператор в $G$. Определим функцию

$$
\Theta(r)= \begin{cases}0, & r<N, \\ f(r), & N \leqslant r \leqslant N+C, \quad \Theta(r) \in C^{2}\left(R_{+}\right) . \\ 1, & r>N+C,\end{cases}
$$

Далее, положим $Q=\Theta \widetilde{U}$. Поскольку $\widetilde{H}(b, V) \widetilde{U}=\lambda \widetilde{U}$, то из (2) следует, что

$$
\widetilde{H}(b, V) Q-\lambda Q=g,
$$

где $g=-\Theta^{\prime \prime} \widetilde{U}-2 \Theta^{\prime} \widetilde{U}^{\prime}$ (здесь и далее штрих означает дифференцирование по $r$ ). Очевидно, что функция $g$ финитна. Введем функцию от переменной $r$ и параметра $s$

$$
\phi_{s}(r)=\frac{1}{s} \operatorname{arctg}(s r)
$$

и определим $Q_{s}=Q e^{\delta \phi_{s}}$. Очевидно, что $Q_{s}$ принадлежит пространству $L_{2}\left(R_{+}, G\right)$ при любых положительных $s$ и $\delta$. Из формул $(2),(3)$ следует, что функция $Q_{s}$ удовлетворяет уравнению

$$
\begin{aligned}
-Q_{s}^{\prime \prime} & -\frac{n-1}{2 r^{2}} Q_{s}+\frac{1}{r^{2}} \sum_{k=1}^{n} D_{k}^{2} Q_{s}+\left[\frac{1}{r} \sum_{k=1}^{n}\left(D_{k} b_{k}+b_{k} D_{k}\right)+b^{2}\right] Q_{s}+ \\
& +V Q_{s}-\lambda Q_{s}+2 \delta \phi_{s}^{\prime} Q_{s}^{\prime}+\left[\delta \phi_{s}^{\prime \prime}-\delta^{2}\left(\phi_{s}^{\prime}\right)^{2}\right] Q_{s}=h_{s}
\end{aligned}
$$


где $h_{s}=g e^{\delta \phi_{s}}$. Умножим уравнение $(4)$ скалярно в $L_{2}\left(R_{+}, G\right)$ на $2 r Q_{s}^{\prime}$ и возьмем вещественную часть, тогда

$$
\begin{aligned}
& \left\|Q_{s}^{\prime}\right\|^{2}-\frac{n-1}{2}\left\|Q_{s}\right\|_{-2}^{2}+\sum_{k=1}^{n}\left\|D_{k} Q_{s}\right\|_{-2}^{2}-\sum_{k=1}^{n}\left(\left[D_{k} b_{k}^{\prime}+b_{k}^{\prime} D_{k}\right] Q_{s}, Q_{s}\right)+ \\
& +\left(b^{2} Q_{s}, Q_{s}\right)-2 \sum_{k=1}^{n}\left(r b_{k} \hat{b}_{k} Q_{s}, Q_{s}\right)+\lambda\left\|Q_{s}\right\|^{2}+4 \delta\left(r \phi_{s}^{\prime} Q_{s}^{\prime}, \phi_{s}^{\prime} Q_{s}^{\prime}\right)- \\
& \quad-\int_{0}^{\infty}\left[r\left(\delta \phi_{s}^{\prime \prime}-\delta^{2}\left(\phi_{s}^{\prime}\right)^{2}\right)\right]^{\prime}\left\|Q_{s}\right\|_{G}^{2} d r=-2 \operatorname{Re}\left(r V Q_{s}, Q_{s}^{\prime}\right)+2 \operatorname{Re}\left(r h_{s}, Q_{s}^{\prime}\right),
\end{aligned}
$$

где $\|.\|_{-2}^{2}=\int_{0}^{\infty} r^{-2}\|\cdot\|_{G}^{2} d r$. Из соотношения (5) следует оценка

$$
\begin{aligned}
& \left\|Q_{s}^{\prime}\right\|^{2}+\sum_{k=1}^{n}\left\|D_{k} Q_{s}\right\|_{-2}^{2}+\lambda\left\|Q_{s}\right\|^{2} \leqslant \varepsilon\left(\sum_{k=1}^{n}\left\|D_{k} Q_{s}\right\|_{-2}^{2}+\left\|Q_{s}\right\|^{2}\right)+ \\
& +\frac{n-1}{2}\left\|Q_{s}\right\|_{-2}^{2}+4\left\|W Q_{s}\right\|^{2}+\frac{1}{4}\left\|Q_{s}^{\prime}\right\|^{2}+4\left\|h_{s}\right\|_{2}^{2}+ \\
& \quad+\frac{1}{4}\left\|Q_{s}^{\prime}\right\|^{2}+\int_{0}^{\infty} \frac{8 \delta r^{2} s^{4}+4 \delta^{2} s^{2} r}{\left(1+s^{2} r^{2}\right)^{3}}\left\|Q_{s}\right\|_{G}^{2} d r
\end{aligned}
$$

где величина $\varepsilon>0$ может быть сделана меньше любого наперед заданного числа путем увеличения $N$ в определении функции $\Theta$, поскольку $\lim _{|x| \rightarrow+\infty}\left|r b_{k}^{\prime}\right|=\lim _{|x| \rightarrow+\infty}\left|b_{k}\right|=$ $0, k=\overline{1, n}$ (см. выше формулы для $b_{k}$ и $b_{k}^{\prime}$ ). Возьмем $\varepsilon$ таким, что $\varepsilon<\min (\lambda / 2,1 / 2)$.

Поскольку для $N>1$ верно неравенство

$$
\frac{n-1}{2}\left\|Q_{s}\right\|_{-2}^{2} \leqslant \frac{n-1}{2 N^{2}}\left\|Q_{s}\right\|^{2}
$$

то $N$ можно выбрать так, что

$$
\frac{n-1}{2}\left\|Q_{s}\right\|_{-2}^{2} \leqslant \frac{\lambda}{4}\left\|Q_{s}\right\|^{2}
$$

Нетрудно показать, что

$$
\frac{8 \delta r^{2} s^{4}}{\left(1+s^{2} r^{2}\right)^{3}} \leqslant s \delta
$$

$$
\frac{4 \delta^{2} s^{2} r}{\left(1+s^{2} r^{2}\right)^{3}} \leqslant \frac{16 \delta^{2}}{27}
$$

а поэтому, выбрав $\delta \leqslant \sqrt{27 \lambda / 128}$ и $s<16 \delta / 27$, можно добиться того, что

$$
\int_{0}^{\infty} \frac{8 \delta r^{2} s^{4}+4 \delta^{2} s^{2} r}{\left(1+s^{2} r^{2}\right)^{3}}\left\|Q_{s}\right\|_{G}^{2} d r<\frac{\lambda}{8}\left\|Q_{s}\right\|^{2} .
$$

С учетом вышесказанного из (6) получим оценку

$$
\left\|Q_{s}^{\prime}\right\|^{2}+\lambda\left\|Q_{s}\right\|^{2} \leqslant \mathrm{C}\left(\left\|W Q_{s}\right\|^{2}+\left\|h_{s}\right\|_{2}^{2}\right) .
$$


Теперь оценим величину $\left\|W Q_{s}\right\|^{2}$. Пусть $R_{0}(-z)=(-\Delta+z)^{-1}, R(-z)=(H(b, V)+$ $z)^{-1}$ - операторы в $L_{2}\left(R^{n}\right)$, тогда $\widetilde{R}(-z)$ - оператор в $L_{2}\left(R_{+}, G\right)$, соответствуюший $R(-z)$. Из теории возмушений и вида $H(b, V)$ в $L_{2}\left(R^{n}\right)$ следует, что

$$
R_{0}(-z)=\left(I+R_{0}(-z)\left(2 \sum_{k=1}^{n} b_{k} p_{k}+b^{2}-i \operatorname{div} b+V\right)\right) R(-z) .
$$

Поскольку норму оператора

$$
R_{0}(-z)\left(2 \sum_{k=1}^{n} b_{k} p_{k}+b^{2}-i \operatorname{div} b+V\right)
$$

в пространстве $L_{2}\left(R^{n}\right)$ для достаточно больших $z>0$ можно оценить сверху единицей, то для какого-либо фиксированного $z_{0}$ оператор

$$
W R\left(-z_{0}\right)=W \sum_{k=0}^{\infty}\left(R_{0}\left(-z_{0}\right)\left(2 \sum_{k=1}^{n} b_{k} p_{k}+b^{2}-i \operatorname{div} b+V\right)\right)^{k} R_{0}\left(-z_{0}\right)
$$

компактен в $L_{2}\left(R^{n}\right)$, следовательно, $W \widetilde{R}\left(-z_{0}\right)$ компактен в $L_{2}\left(R_{+}, G\right)$. Из $(4)$ следует, чTO

$$
W Q_{s}=W \widetilde{R}\left(-z_{0}\right)\left(\left(\lambda+z_{0}\right) Q_{s}-2 \delta \phi_{s}^{\prime} Q_{s}^{\prime}-\left[\delta \phi_{s}^{\prime \prime}-\delta^{2}\left(\phi_{s}^{\prime}\right)^{2}\right] Q_{s}+h_{s}\right) .
$$

Оценим результат действия оператора $W \widetilde{R}\left(-z_{0}\right)$ на первое слагаемое в правой части (8). В силу компактности оператора $W \widetilde{R}\left(-z_{0}\right)$ для любого $\varepsilon_{1}$ сушествуют числа $N_{1}\left(\varepsilon_{1}\right)$ и $C$ такие, что

$$
\left\|W \widetilde{R}\left(-z_{0}\right)\left(\lambda+z_{0}\right) Q_{s}\right\| \leqslant \varepsilon_{1}\left\|Q_{s}\right\|+C\left(\int_{0}^{N_{1}}\left\|Q_{s}\right\|_{G}^{2} d r\right)^{\frac{1}{2}} .
$$

При оценке остальных слагаемых правой части (8) оператор $W \widetilde{R}\left(-z_{0}\right)$ можно считать просто ограниченным. Тогда из соотношения (8) и ограниченности функций $\phi_{s}^{\prime}$ и $\phi_{s}^{\prime \prime}$ вытекает оценка

$$
\left\|W Q_{s}\right\|^{2} \leqslant C\left(\varepsilon_{1}^{2}\left\|Q_{s}\right\|^{2}+\int_{0}^{N_{1}}\left\|Q_{s}\right\|_{G}^{2} d r+\delta^{2}\left\|Q_{s}^{\prime}\right\|^{2}+\left(\delta+\delta^{2}\right)^{2}\left\|Q_{s}\right\|^{2}+\left\|h_{s}\right\|^{2}\right) .
$$

Числа $\varepsilon_{1}$ и $\delta$ можно взять настолько малыми, что $C\left(\varepsilon_{1}^{2}+\left(\delta+\delta^{2}\right)^{2}\right)<\lambda$ и $C \delta^{2}<1$. Тогда, применив неравенство (9) для оценки правой части $(7)$, получим

$$
\left\|Q_{s}\right\|^{2} \leqslant C\left(\left\|h_{s}\right\|_{2}^{2}+\int_{0}^{N_{1}}\left\|Q_{s}\right\|_{G}^{2} d r\right),
$$

откуда в силу финитности $h_{s}$ следует, что $\left\|Q_{s}\right\| \leqslant C$ для любого $s>0$, а поэтому

$$
e^{\delta_{0}|x|} U(x) \in L_{2}\left(R^{n}\right)
$$

для какого-то $\delta_{0}>0$. Остается применить теорему 2 из $[1]$, в силу которой $U(x) \equiv 0$. Теорема 1 доказана. 


\section{3. АБСОЛЮТНАЯ НЕПРЕРЫВНОСТЬ СПЕКТРА}

Теорема 2. Пусть выполнены следующие условия:

a) $\left|\hat{b}_{k}(x)\right|=o\left(\frac{1}{|x|}\right), \quad|x| \rightarrow \infty, \sup _{x \in R^{n}}\left|\hat{b}_{k}(x)\right|<\infty, \quad k=\overline{1, n}$;

б) $\sup _{x \in R^{n}}|\operatorname{div} b(x)|<\infty$;

в) при $n \leqslant 3$ оператор $W=V(1+|x|) \Delta$-компактен;

г) при $n>3$ оператор $W \Delta$-компактен $u$

$$
\lim _{z \rightarrow+\infty} z^{\frac{1}{4}}\left\|(1+|x|)^{-\frac{1}{2}} W(-\Delta+z)^{-1}\right\|=0 ;
$$

д) оператор $|x| b^{2} \Delta$-компактен.

Тогда оператор (1) обладает абсолютно непрерывныц спектром на положительной полуоси.

ДокАЗАТЕЛЬСтво. Используем оценку Мурра (см., например, [3], с. 84). Оператор $H(b, V)$ удовлетворяет этой оценке на интервале $\psi$, если сушествуют положительное число $\alpha$ и компактный оператор $K$ такие, что $E_{\psi}[H(b, V), i A] E_{\psi} \geqslant \alpha E_{\psi}+K$. Здесь $E_{\psi}-$ спектральньй проектор $H(b, V)$, отвечающий интервалу $\psi,[H(b, V), i A]=H(b, V) i A-$ $i A H(b, V)$ - коммутатор. В нашем случае в качестве оператора $A$ можно взять $(x \cdot \nabla+$ $\nabla \cdot x) /(2 i)$. Вычисляя, получаем выражение

$$
E_{\psi}[H(b, V), i A] E_{\psi}=2 E_{\psi} H(b, V) E_{\psi}+E_{\psi} T E_{\psi},
$$

где

$$
\begin{aligned}
T= & {\left[2 i \operatorname{div} b+2 i \sum_{k=1}^{n} b_{k} \frac{\partial}{\partial x_{k}}-2 b^{2}-2 V+\right.} \\
& \left.+2 i \sum_{k=1}^{n}\left(\sum_{m=1}^{n} x_{m} \frac{\partial b_{k}}{\partial x_{m}}\right) \frac{\partial}{\partial x_{k}}+i \sum_{k=1}^{n} \sum_{m=1}^{n} x_{m} \frac{\partial^{2} b_{k}}{\partial x_{m} \partial x_{k}}-|x| \frac{\partial\left(b^{2}+V\right)}{\partial|x|}\right] .
\end{aligned}
$$

Пусть $\psi=(\alpha, \beta), \alpha>0$. Поскольку $E_{\psi} H(b, V) E_{\psi} \geqslant \alpha E_{\psi}$, то остается показать компактность второго слагаемого правой части (10). Так как

$$
\begin{aligned}
E_{\psi} T E_{\psi}= & E_{\psi}(H(b, V)+z)(H(b, V)+z)^{-1}(-\Delta+z)(-\Delta+z)^{-1} \times \\
& \times T(-\Delta+z)^{-1}(-\Delta+z)(H(b, V)+z)^{-1}(H(b, V)+z) E_{\psi}
\end{aligned}
$$

(для подходяших $z>0$ ) и операторы

$$
\begin{aligned}
E_{\psi}(H(b, V)+z), & (H(b, V)+z) E_{\psi}, \\
(H(b, V)+z)^{-1}(-\Delta+z), & (-\Delta+z)(H(b, V)+z)^{-1}
\end{aligned}
$$

ограничены (первые два в силу определения $E_{\psi}$, два оставшихся в силу представления для $R(-z)=(H(b, V)+z)^{-1}$, полученного в разделе 1$)$, то достаточно доказать компактность

$$
(-\Delta+z)^{-1} T(-\Delta+z)^{-1} .
$$


Компактность этого выражения следует из компактности отдельных его слагаемых. Действительно, пусть $K(x, y,-z)$ - ядро оператора $R_{0}(-z) \operatorname{div} b R_{0}(-z)$. Тогда

$$
K(x, y,-z)=\int_{R^{n}} R_{0}(x, s,-z) \operatorname{div} b(s) R_{0}(s, y,-z) d s .
$$

Интегрируя по частям и учитывая то, что $\lim _{|x| \rightarrow \infty}\left|b_{k}\right|=0, k=\overline{1, n}$, получим

$$
\begin{aligned}
K(x, y,-z)= & \int_{R^{n}} \sum_{k=1}^{n} \frac{\partial R_{0}(x, s,-z)}{\partial x_{k}} b_{k} R_{0}(x, s,-z) d s+ \\
& +\int_{R^{n}} \sum_{k=1}^{n} R_{0}(s, y,-z) b_{k}(s) \frac{\partial R_{0}(s, y,-z)}{\partial y_{k}} d s
\end{aligned}
$$

т.e.

$$
R_{0}(-z) \operatorname{div} b R_{0}(-z)=\sum_{k=1}^{n}\left(\frac{\partial}{\partial x_{k}} R_{0}(-z) b_{k} R_{0}(-z)-R_{0}(-z) b_{k} R_{0}(-z) \frac{\partial}{\partial y_{k}}\right)
$$

Этот оператор компактен, так как операторы $\frac{\partial}{\partial x_{k}} R_{0}(-z)$ и $R_{0}(-z) \frac{\partial}{\partial y_{k}}$ ограничены, а $R_{0}(-z) b_{k}$ и $b_{k} R_{0}(-z)$ компактны, поскольку, как уже отмечалось, $\lim _{|x| \rightarrow+\infty} b_{k}(x)=0$, $k=\overline{1, n}$.

Операторы

$$
\sum_{k=1}^{n} R_{0}(-z) b_{k} \frac{\partial}{\partial x_{k}} R_{0}(-z), \quad R_{0}(-z)\left(b^{2}+V\right) R_{0}(-z)
$$

и

$$
\sum_{k=1}^{n} \sum_{m=1}^{n} R_{0}(-z)\left(x_{m} \frac{\partial b_{k}}{\partial x_{m}}\right) \frac{\partial}{\partial x_{k}} R_{0}(-z)
$$

также компактны, так как оператор $\frac{\partial}{\partial x_{k}} R_{0}(-z)$ ограничен,

$$
\lim _{|x| \rightarrow+\infty} b_{k}(x)=0, \quad \sum_{m=1}^{n} x_{m} \frac{\partial b_{k}}{\partial x_{m}}=\frac{\partial b_{k}}{\partial|x|}, \quad \lim _{|x| \rightarrow+\infty}|x| \frac{\partial b_{k}}{\partial|x|}=0, \quad k=\overline{1, n}
$$

и оператор $V \Delta$-компактен.

Интегрируя по частям ядро оператора, получим

$$
\begin{aligned}
\sum_{k=1}^{n} & \sum_{m=1}^{n} R_{0}(-z)\left(x_{m} \frac{\partial^{2} b_{k}}{\partial x_{m} \partial x_{k}}\right) R_{0}(-z)= \\
= & \sum_{k=1}^{n} \sum_{m=1}^{n} \frac{\partial}{\partial x_{k}} R_{0}(-z)\left(x_{m} \frac{\partial b_{k}}{\partial x_{m}}\right) R_{0}(-z)- \\
& -\sum_{k=1}^{n} \sum_{m=1}^{n} R_{0}(-z)\left(x_{m} \frac{\partial b_{k}}{\partial x_{m}}\right) R_{0}(-z) \frac{\partial}{\partial y_{k}}
\end{aligned}
$$

4 Теоретическая и математическая физика, т. 130, № 2, 2002 г. 
Очевидно, что этот оператор компактен.

Также путем интегрирования по частям ядра оператора найдем, что

$$
\begin{aligned}
& R_{0}(-z)|x| \frac{\partial\left(b^{2}+V\right)}{\partial|x|} R_{0}(-z)=-n R_{0}(-z)\left(b^{2}+V\right) R_{0}(-z)+ \\
& \quad+\frac{\partial}{\partial|x|} R_{0}(-z)|x|\left(b^{2}+V\right) R_{0}(-z)-R_{0}(-z)|x|\left(b^{2}+V\right) R_{0}(-z) \frac{\partial}{\partial|y|}
\end{aligned}
$$

Этот оператор также компактен, в силу того что операторы $\frac{\partial}{\partial|x|} R_{0}(-z)$ и $R_{0}(-z) \frac{\partial}{\partial|y|}$ ограничены, а $|x| b^{2}$ и $|x| V \Delta$-компактны по условиям теоремы 2.

Таким образом, доказано, что оператор (1) удовлетворяет оценке Мурра на положительной полуоси, следовательно, у него на этой полуоси отсутствует сингулярно-непрерывный спектр ([3], с. 90). А поскольку условия теоремы 2 включают условия теоремы 1 , то у оператора (1) на интервале $(0, \infty)$ отсутствуют собственные значения. Следовательно, оператор (1) обладает абсолютно непрерывным спектром на положительной полуоси. Теорема 2 доказана.

Благодарности. Автор благодарит Х. Х. Муртазина за поставленную задачу.

\section{Список литературы}

[1] М. Б. Губайдуллин, Х. Х. Муртазин. ТМФ. 2001. Т. 126. № 3. С. 443.

[2] X.X. Муртазин, В.А. Садовничий. Спектральный анализ многочастичного оператора Шредингера. М.: Изд-во МГУ, 1988.

[3] Х. Цикон, Р. Фрёзе, В. Кири, Б. Саймон. Операторы Шредингера с приложениями в квантовой механике и глобальной геометрии. М.: Мир, 1990.

Поступила в редакцию 2.VIII.2001 г. 